\title{
FUTURE ASTROMETRY FROM SPACE AND FROM THE GROUND
}

\author{
E. H $\phi$ g and H.J. Fogh 01sen \\ Copenhagen University Observatory, Denmark
}

\section{ABSTRACT}

The space astrometry projects being studied by the European space Agency (ESA) promise an accuracy about \pm 0.003 due to photon statistics for about 100000 stars. Inherent limitations of the space astrometry projects and the future continued importance of ground based astrometry are outlined.

\section{INTRODUCT ION}

Current and planned developments of new astrometric instrumentation carry great promise for this part of astronomy which provides basic data of stellar positions, distances, masses and stellar velocities. The developments concern many fields: photographic astrometry with reflectors and astrographs, meridian astrometry, radio astrometry and space astrometry. The first part of the following presentation is concerned with space astrometry. The second part shall discuss the limitations of space astrometry and its relation to ground based astrometry.

\section{SPACE ASTROMETRY}

ESA has carried out a Mission Definition Study of Space Astrometry. This study appeared in 1976 and presents three possibilities or options of space instruments: two options suitable for launch by the Scout launcher, i.e. a rather small rocket, and one option for spacelab.

The basic limitations of ground based astrometry compared to space astrometry are due to image motion and refraction anomalies caused by the atmosphere and due to bending of the meridian circle telescope under gravity. Although significant improvement of ground based astrometry is still possible, the expected improvement through space astropetry is orders of magnitude greater with respect to accuracy and to the number of stars.

The role of astrometry in the scientific framework of astronomy is to provide fundamental data complementary to other astrophysical data. 
The cosmic distance scale also to the external galaxies is built up as a sequence of calibrations, which in the first steps rely on the geometric determination of distances to the nearby stars. Here are indispensable the trigonometric parallaxes of nearby stars and the peoper motions of the Hyades, which in turn give the stream parallax of the nearby cluster at $42 \mathrm{pc}$.

Our direct knowledge of stellar masses below that of the solar mass relies on a knowledge of the trigonometric parallaxes of binary stars.

In the context of galactic structure astrometry provides data on space velocities of stars which cannot be obtained by other means. Space velocities are derived from proper motions, radial velocities and photometric distances (calibrated as mentioned above). Studies of the gravitational potential of the Galaxy, of the parameters of galactic rotation and of stellar motion, of birth places of young stars and of the distribution of space velocities for different classes of stars can be made. Study of gradients in the velocity distribution of stars related to spiral arms will be possible and provide test of the theory

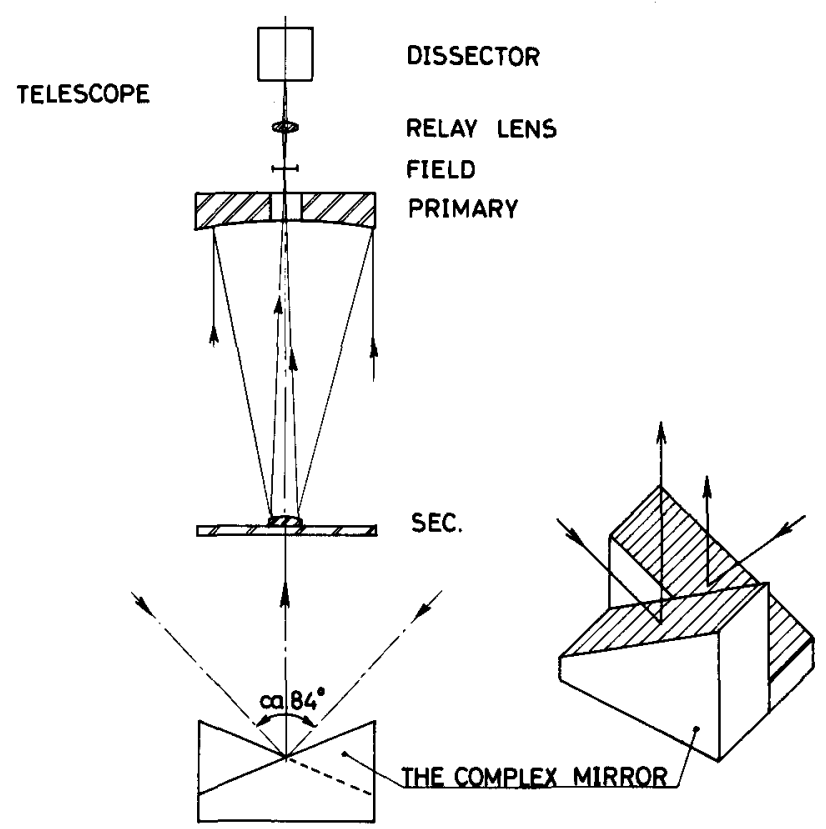

Figure 1. Optical system of option A. 
of density waves. Membership of stars in clusters can be studied. All of this would greatly profit from a space astrometry mission.

The three technical options ESA has studied for performing astrometry from space all rely on the measurement of angles between stars by means of a telescope forming a superposed image of two fields of the sky separated by about $90^{\circ}$ on the sky, a technique originally proposed by Prof. Lacroute.

The basic problem is to overcome both short- and long-term attitude variations of the three-axis stabilized satellite.

I shall now for the sake of clarity and brevity describe the option $A$ in some detail and afterwards the option B and the Spacelab option.

The optical system of option A is shown in Fig. 1. The complex mirror consists of two plane mirrors that form a very constant angle about $45^{\circ}$ with each other. A Schmidt-Cassegrain telescope of $16 \mathrm{~cm} \mathrm{x}$ $16 \mathrm{~cm}$ aperture forms the image of the two fields of about one square degree area.

The satellite is launched in a polar six-hour sun-synchronous orbit, as the TDl satellite. It rotates uniformly once per orbit so that the two optical axes scan the same great circle, one axis following the other. The rotation axis is actively stabilized by means of a fixed momentum wheel with its axis parallel to the rotation axis of the satellite. A gyro shall provide instantaneous attitude information around this axis. In connection with a star mapper in the field of the telescope (Fig. 2) accurate absolute real-time attitude reconstitution is provided so that preselected stars can be observed according to a list.

A star moves across a system of slits about 1" wide, the diffraction limited size of a star image. The active cathode spot of an image dissector is moved along with the star according to the accurate attitude knowledge. Photon counting is performed in sampling intervals of $1 \mathrm{~ms}$ timed by a good quartz clock.

The angle between two stars in the field can be derived from the transit times at the slits if the cathode spot is made to follow first one star (during $0.2 \mathrm{~s}$ ) and then the other star. The spot must be switched several times between the two stars in order that the disturbing attitude jitter be reduced in the difference between the mean values of the transit times.

After a complete rotation of $360^{\circ}$, i.e. after one orbit, most of the previous stars on the great circle will appear again, and this provides the fundamental technique to calibrate both the $90^{\circ}$ angle between the two axes and the scale value of the telescope at the slit system. 


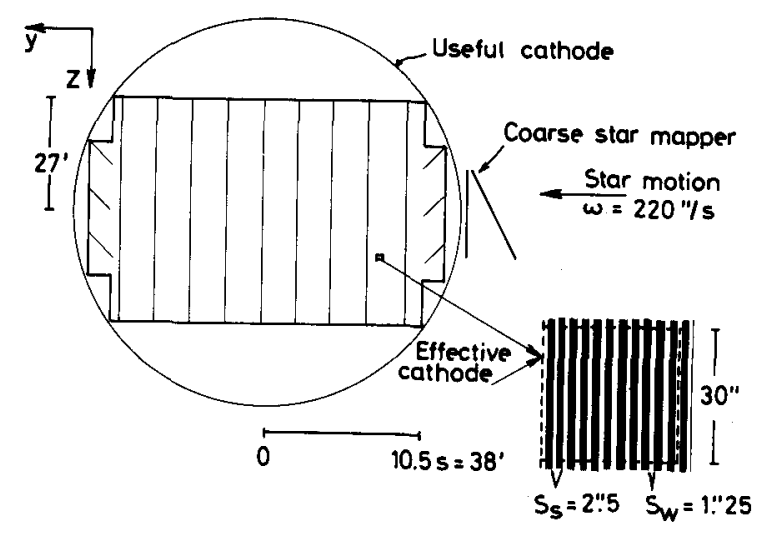

Figure 2. Field of view of image dissector, option A.

Time is too short here to explain how stars on the whole sky can be connected by way of the measurement along a system of intersecting great circles. It is important to state, however, that the numerical separation of the five astrometric unknowns of a star $\pi, \lambda, \beta, \mu_{\lambda}$, and $\mu_{\beta}$ requires observations distributed on at least five epochs of about three years.

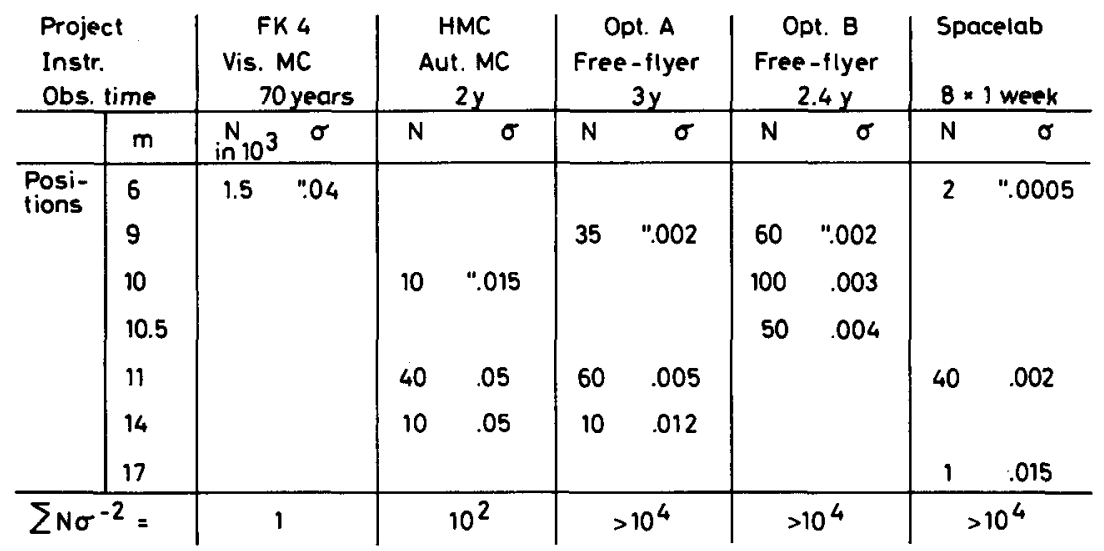

The Table shows the expected performance of the three space astrometry options compared with the presently best observed stars, the 1500 stars of FK4. Space astrometry provides 20 times smaller errors of positions for 100 times as many stars and for 100 times as faint stars. The optimistically estimated progress by means of a HMC (horizontal meridian circle) is considerable, but far below that af space astrometry. 
The option B gives a similar performance as far as photon statistics are concerned; the only error source in fact counted in the Table for the three space astrometry proposals. It is the design goal to keep other sources of error below 0".001. With option B all stars brighter than a fixed limiting magnitude $\left(\mathrm{m}_{\mathrm{pg}}=10.5\right)$ are observed during equally long integration times, with $\mathrm{pg}_{\mathrm{c}}$ advantage that no accurate attitude knowledge is required in real time. The advantage of the preselection foreseen with option A gives the possibility to concentrate five times as long integration times on a selected star than with option $B$, in order to improve the accuracy. The stars for option A, for instance 50000 stars, should be selected according to their astrophysical interest, and other stars as optimal, representative samples for an unbiased study of galactic structure.

Another feature of option $B$ is to apply passive, gravity gradient stabilization, thus, obtaining a more smooth attitude motion, with the reward that a smaller field of view is sufficient and therefore a simpler optical system of Ritchey-Chrétien type.

The Spacelab option gives good performance especially on faint stars. It was the preferred option before the mission definition study started, but it lost that position when we were able to improve the light economy of the original TD astrometry project by a hundred times and at the same time accommodate it in a Scout launcher.

\section{ASTROMETRY FROM SPACE AND FROM GROUND}

In view of the great scientific significance of future successful space astrometry it is important also to point out its limitations, and to show the areas where ground based astrometry remains indispensable. It must also be realized that already the expectations to space astrometry seem to have repercussions on the planning of ground based astrometry even before we know whether space astrometry is feasible. This is a particular risk for science since first-class astrometry today is carried by so few institutions that we can miss none of them without endangering both space astrometry and ground based astrometry.

Such a discussion must of course be based on the actually proposed space astrometry projects, since with no instrumental or financial constraints one might imagine to do everything better from space, being free of disturbances from the atmosphere and from gravity. Such ideal performance is implied in some of the recent predictions for space astrometry, e.g. in the all too optimistic statement that "Space astrometry will have no systematic errors".

The present discussion shall be limited to the ESA space astrometry projects, which are liqted in the Table. The planned astrometry from NASA Space Telescope (ST) covers quite different fields: only small angles; high angular resolutions; faint stars; few objects. Therefore, 
it can observe binaries, relative parallaxes and relative proper motions. The ESA options (of which not more than one will be realized) are designed to connect many stars on the sky by angular measurements to form a rigid system of positions and proper motions (reference system) as required for the study of galactic structure etc.

Any of the three ESA space missions lasting about 3 years are expected to provide for bright stars individual parallaxes with a mean error $\pm 0: 003$, positions $\pm 0 ": 002$ and proper motions $\pm 0 ": 002$ per year. These accidental errors are expected from photon statistics, whereas the design goal for systematic and other errors are to be below \pm 0 ":001.

Such achievements would have enormous impact on our knowledge of the cosmic distance scale, of the stellar masses, of galactic structure and of geodynamics. But since this has often been pointed out, it is perhaps more urgent to list the technical limitations of the proposed space projects:

1. point sources only, diameter < $0 " 5$,

2. Fixed elongations from Sun, about $\pm 90^{\circ}$,

3. three years of observation with five epochs only,

4. rigid, but rotating coordinate system,

5. fixed and limited number of stars,

6. limiting magnitude,

7. feasibility not yet ascertained,

8. high cost.

These, mainly technica1, limitations and how they interact to produce scientific limitations shall be explained.

The limitations are valid for all three options, except that the Spacelab option may observe quasars directly and thereby determine geometrically the rotation of the system, since quasars have no proper motions.

The limited observing time of three years makes the dynamical determination of the rotation of the system by way of minor planets hardly possible, since the orbital elements of planets and Earth cannot be separated in so short time. The limitation to fixed elongations of $90^{\circ}$ and only five epochs makes this even worse. The Spacelab option is not 1 imited to fixed elongations, but the high cost of each spacelab mission will restrict the number of missions to the absolute minimum of about six.

On the other hand, the rotation of the system can be well determined from the ground. Meridian observations of major and minor planets can be much more accurate with a photoelectric multislit micrometer than with the classical visual micrometers. Also an accurate geometrical determination of the inertial system from the ground is feasible in two steps: Firstly, observe by photographic astrometry the proper motion of faint quasars relative to stars of $\mathrm{m}=14$ around each quasar. Secondly, determine the proper motions of the stars from space (option A). 
Important fields of research for accurate meridian circles, if space astrometry becomes successful, are the following. Observe orbits of major and minor planets and of Sun and Moon; dynamic determination of inertial system from these observations; geometric determination of inertial system by bridging a magnitude gap from $m=10.5$ (option $B$ ) to $m=13$; check the reference system provided by space astrometry; tie further astrophysically interesting stars not included in the original space observations into the system; tie further reference stars into the space reference net as required by photographic astrometry either for special purposes in limited areas or for a denser general purpose net; provide second epoch observations to be combined with the first epoch positions obtained from space and thus improve proper motions. An asymptotic mean error of \pm 0 ":01 for relative meridian positions of stars if expected.

The important fields of research for photographic astrometry in case of successful space astrometry derive from the unchallenged storage capacity of the photographic plate and from its ability to integrate the light from fainter star, thus providing parallaxes, positions and proper motions of fainter stars and of many more stars.

The time scale of space astrometry with the probable ESA budget points at a launch in 1985 and astrometric results in 1990. Before the results are on ground nobody could be perfectly certain of the success. This uncertainty, the high cost of space astrometry and the other above mentioned limitations must urge us to continue our best efforts from ground.

\section{REFERENCES ON SPACE ASTROMETRY}

1. Space Astrometry, Proceedings of a Symposium held at Frascati, document ESRO-SP-108, March 1975.

2. Space Astrometry, Report on the Mission Definition Study, document ESA-DP/PS(76)11, Neuil1y 19 May 1976.

3. New Problems in Astrometry, IAU, Symposium No. 61, ed. by W. Gliese, C.A. Murray, and R.H. Tucker, 1974, p. 277 and p. 283. 


\section{DISCUSSION}

C.A. MURRAY: We seem to have been given two very different estimations for the accuracy for parallaxes.

E. HØG: In option A the limitations by photons give 0!003 for $m=9$ and $0: 0004$ for $m=6$. But the accuracy may not be better than $0 ! 001$ in any case.

W. DIECKVOSS: Are there problems in knowing which stars are observed?

E. HOG: Yes. The stars have to be identified in any case, but in different way for option $A$ and option $B$.

H.G. WALTER: The large telescope project, as well as ESA options A and $B$ requires reference stars. Which are these reference stars and how accurate must their positions be known?

P. LACROUTE: In option $A$ we do not need any previous positions. In option $B$ approximate positions for many stars are good enough.

E. $H \emptyset \mathrm{G}$ : It is important to involve more people in space astrometry. Those interested can obtain a report from ESA. DP/PS(76)11.

C.A. MURRAY: It is important to do space astrometry but how are we going to organize it? Can any single institute undertake it and what should be the role of I.A.U. commissions 8 and 24 , because these are responsible for providing good data.

W. van ALTENA: This has been discussed in NASA with regard to the LST. All observations may be derived from individual proposals which will get observing time on the telescope LST; results will be made available to everybody.

E. $H \emptyset \mathrm{G}$ : Dr. Manno, how is it for ESA?

DR. MANNO: It remains to be discussed.

P. BROSCHE: Astrophysics can be organized as individual projects, but

it is different for astrometry.

C.A. MURRAY: ESA provides data as I understand it; but who is going to provide the results unless we get a large group working on it. I suggest that a few members of commissions 8 and 24 discuss this problem.

VAN HERK: Does anyone volunteer for this?

J. ANDERSEN: Will a feasibility study from ESA include a study of the possibility of getting through with the reductions, and you must also know in advance which stars you are going to observe. 
C.A. MURRAY: Should we perhaps set up a working group?

E. $H \varnothing G$ : Maybe it will be better if an existing institution such as ESO organize the project.

VAN HERK: In conclusion now we hope Murray and Hфg will keep this under discussion.

A resolution is proposed by $P$. Lacroute. The text of the resolution in favor of space astrometry is included in the report of commission 8 and is accepted by commissions 8 and 24 .

W. van ALTENA: ST and ESA projects are complementary and I support the resolution. 